\title{
Adaptive Multi-Scale Feature Fusion Based Residual U-net for Fracture Segmentation in Coal Rock Images
}

\section{CURRENT STATUS: UNDER REVIEW}

EURASIP Journal on Image and Video Processing Springer

Fengli Lu

China university of Ming and Technology(Beijing)

Chengcai Fu

China Universtity of Ming and Technology(Beijing)

Guoying Zhang

zhangguoying1101@163.comCorresponding Author

ORCiD: https://orcid.org/0000-0002-6674-1632

Jie Shi

China University of Ming and Technology(Beijing)

DOI:

$10.21203 / \mathrm{rs} .2 .23959 / \mathrm{v} 1$

\section{SUBJECT AREAS}

Publishing/Media

\section{KEYWORDS}

adaptive multi-scale feature fusion, $U$-net, fracture segmentation in coal rock CT images, dilation convolutions, residual U-net 


\section{Abstract}

Accurate segmentation of fractures in coal rock CT images is important for safe production and the development of coalbed methane. However,to make segment coal rock fractures accurate, the challenges as the following:1)The coal rock CT images have the characteristics which are high background noise, sparse target, weak boundary information, uneven gray level, low contrast etc.; 2)There is no a public dataset of coal rock CT images;3)Limited coal rock CT images samples.In the paper,we proposed adaptive multi-scale feature fusion based residual U-uet(AMSFFRU-uet) for fracture segmentation in coal rock CT images to address the issues.In order to reduce the loss of tiny and weak fractures, dilated residual blocks (DResBlock) are embedded into the U-uet structure, which expand the receptive field and extract fracture information atdifferent scales.Furthermore, for reducing the loss of spatial information during the down-sampling process, feature maps of different sizes in the encoding branch are concatenated by adaptive multi-scale featurefusion module, which is as the input of the first up-sampling in the decoding branch.And we applieda set of comprehensive data augmentation operations to increase the diversity of training samples. Our network,U-net and ResU-net are tested on our dataset of coal rock CT images with 5 different textures. The experimental results show that compared with U-net and ResU-net, our proposed approach improve the average Dice coefficient by $5.1 \%$ and $2.9 \%$ and the average accuracy by $4.5 \%$ and $2 \%$, respectively. Therefore,AMSFFRU-net can achieve better segmentation of coal rock fractures, and has stronger generalization ability and robustness.

\section{Full Text}

Due to technical limitations, full-text HTML conversion of this manuscript could not be completed. However, the manuscript can be downloaded and accessed as a PDF.

\section{Figures}




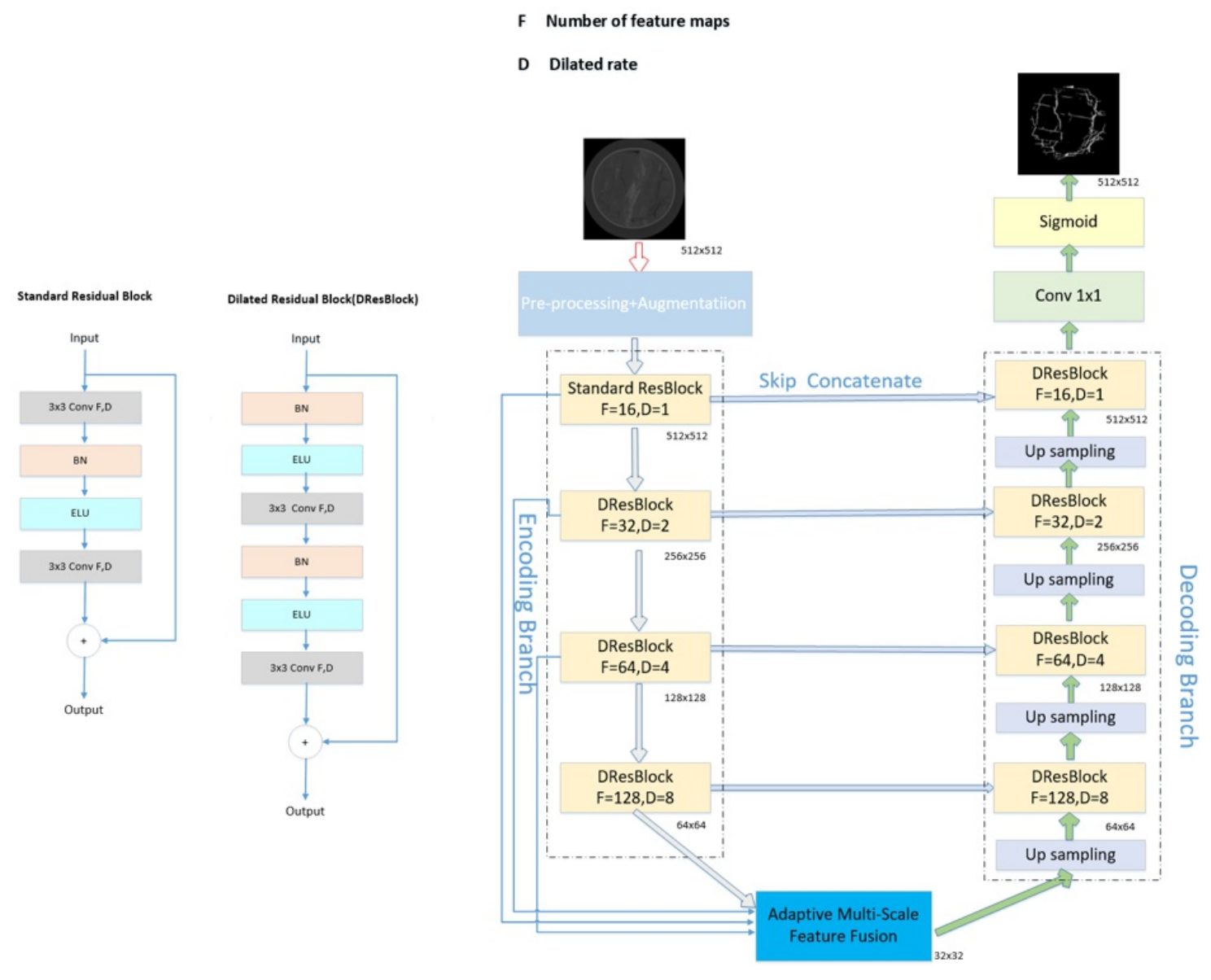

Figure 1

Architecture of the proposed AMSFFRU-net, which comprises encoding branch,AMSFF and decoding branch.1) Encoding branch consisted of one standard residual block with a dilation rate of 1 and three dilated residual block(DResBlock) with dilation rates(2,4,8). 2)AMSFF adaptive fuse the different scales feature maps of all cell blocks from encoding branch.3) Decoding branch consisted of one standard block with a dilation rate of 1 , three dilated residual block(DResBlock) with dilation rates(2,4,8) and 4 up-sampling layers. 4)The number below each rectangle represents the size of the feature map, such as $256 \times 256$. 


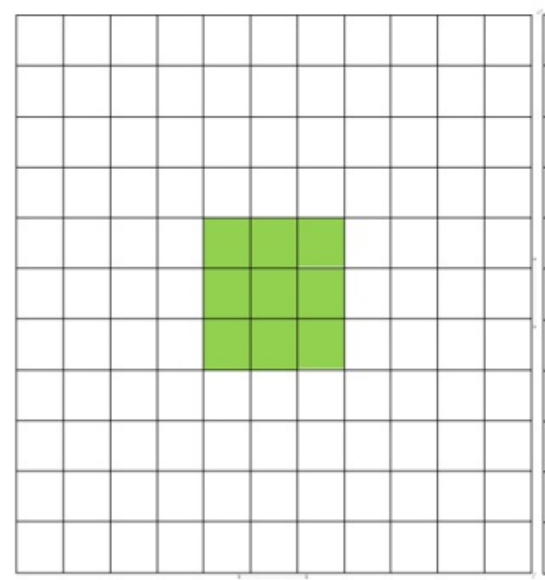

(a)

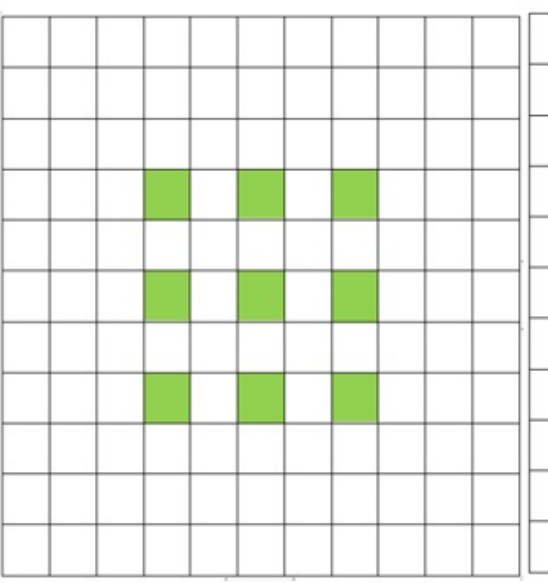

(b)

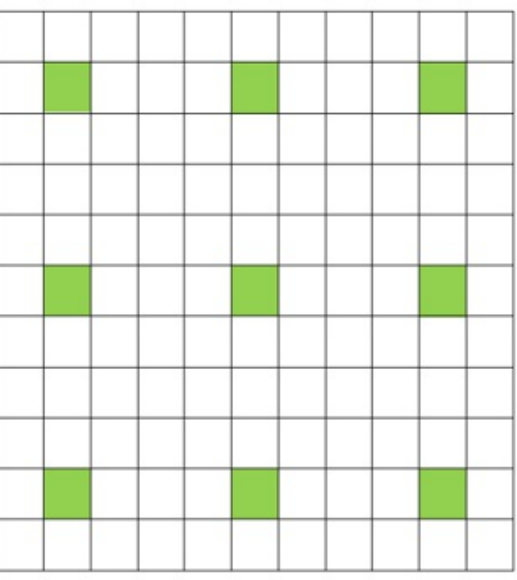

(c)

Figure 2

Illustration of dilated Convolution kernels size with different dilated rates.(a)dilated rate $=1$;

(b) dilated rate $=2$; (c) dilated rate $=4$. 
Standard Residual Block

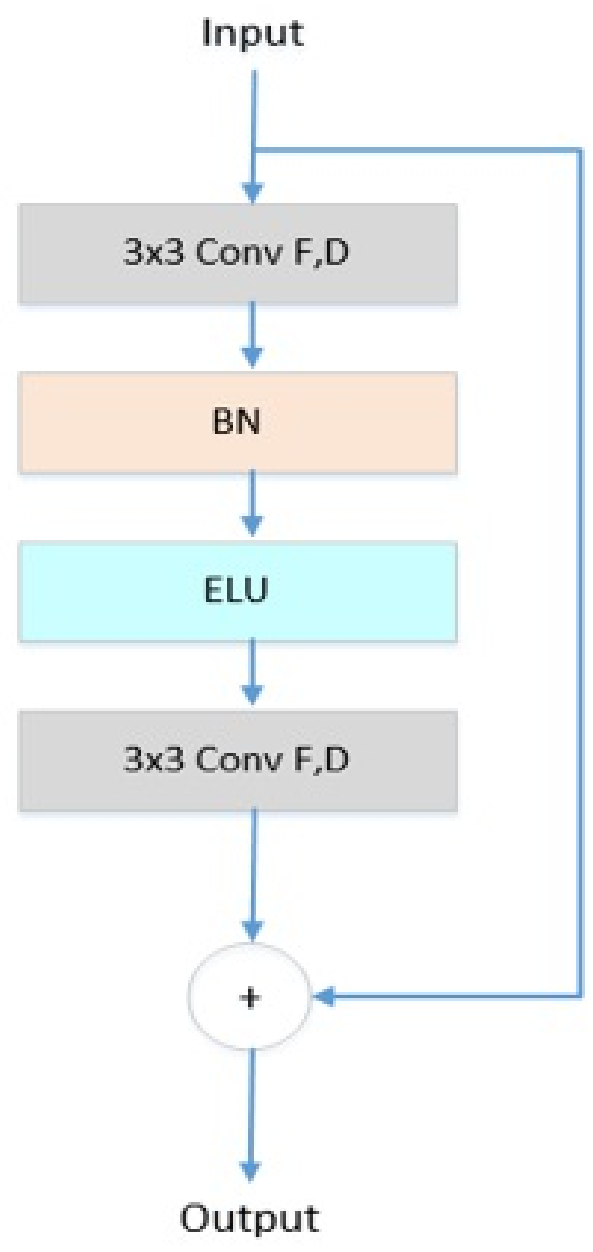

(a)
Dilated Residual Block(DResBlock)

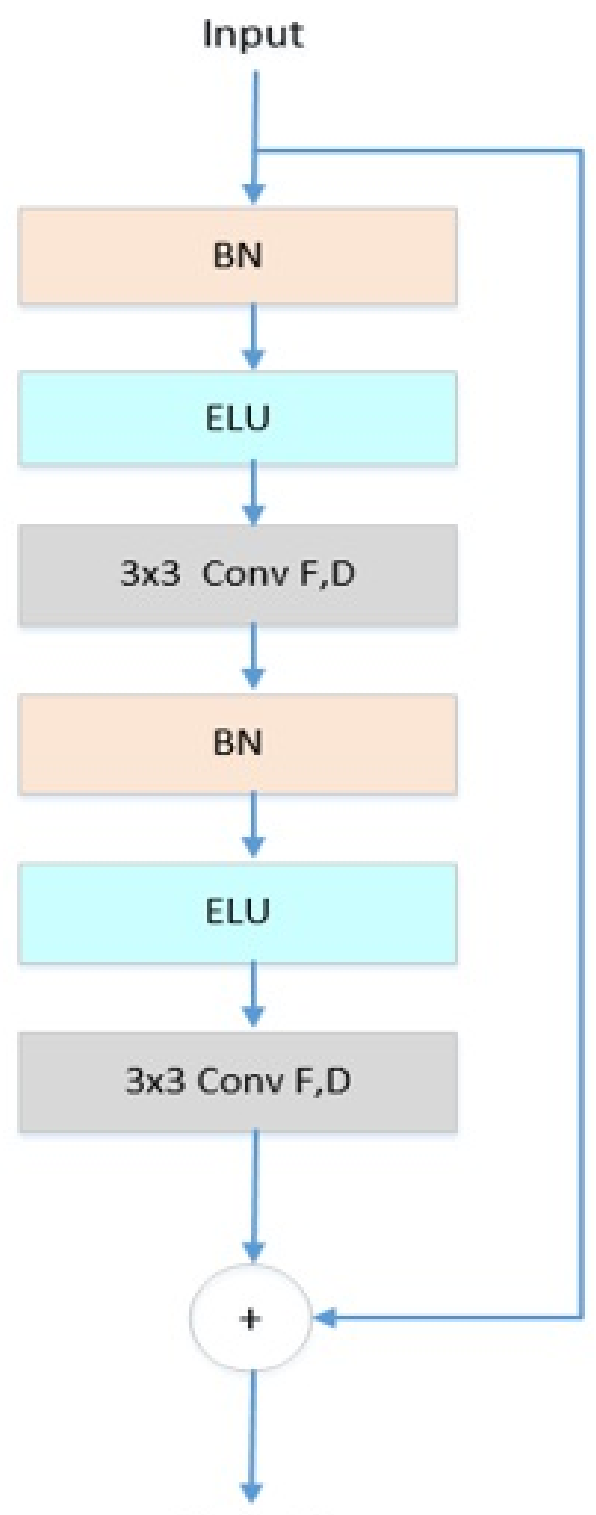

Output

\section{(b)}

Figure 3

Illustration of the structure of dilated residual block.(a)The standard residual block, $D=1$.(b)It is deformed residual block, the value of $D$ is different for different layers. 


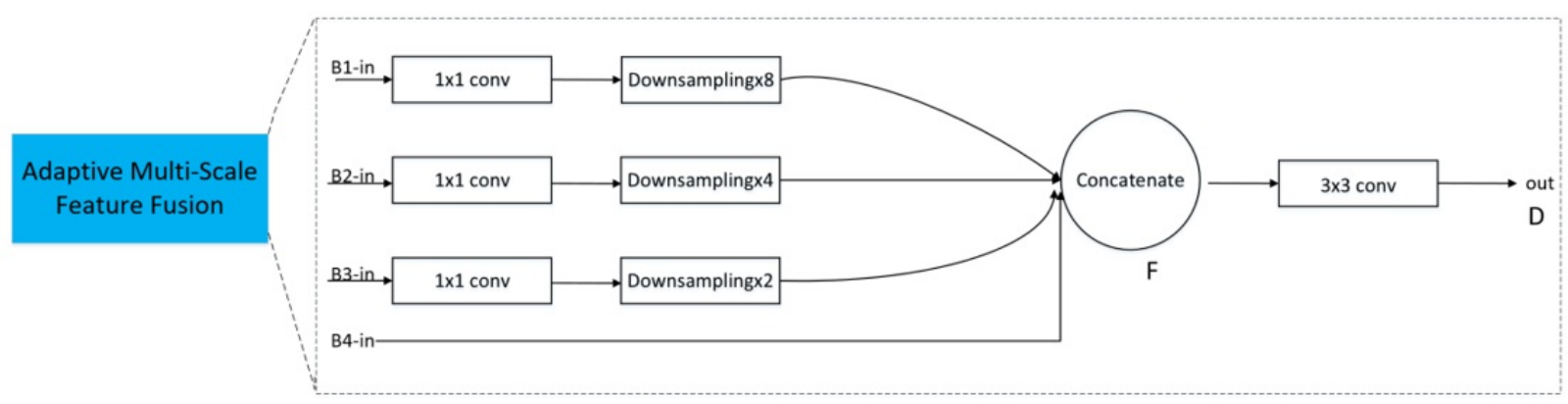

Figure 4

Structure of AMSFF: 1)The input feature $\operatorname{maps}(,$, , ) undergo a $1 \times 1$ convolution layer to reduce parameter calculation,firstly; then they reduce the dimension by convolutions with strides 2 to obtain the same size. 2)The input feature map directly connected because of its dimensions are the same as the feature map . 3)The merged feature map undergo a $3 \times 3$ convolution as the input of decoding branch. 


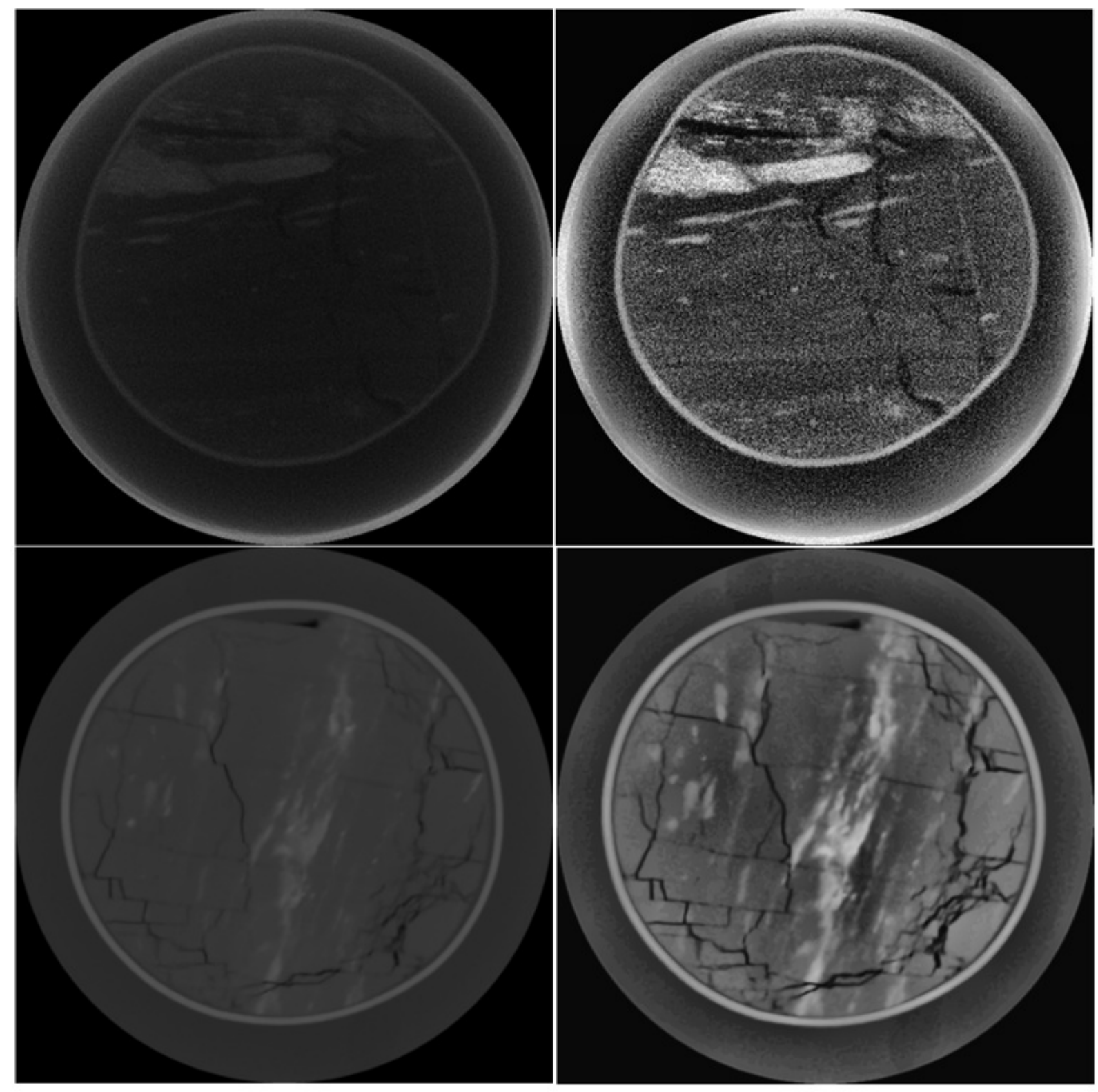

(a)

(b)

Figure 5

The result image after preprocessing: (a)original image.(b) after globally CLAHE operation. 

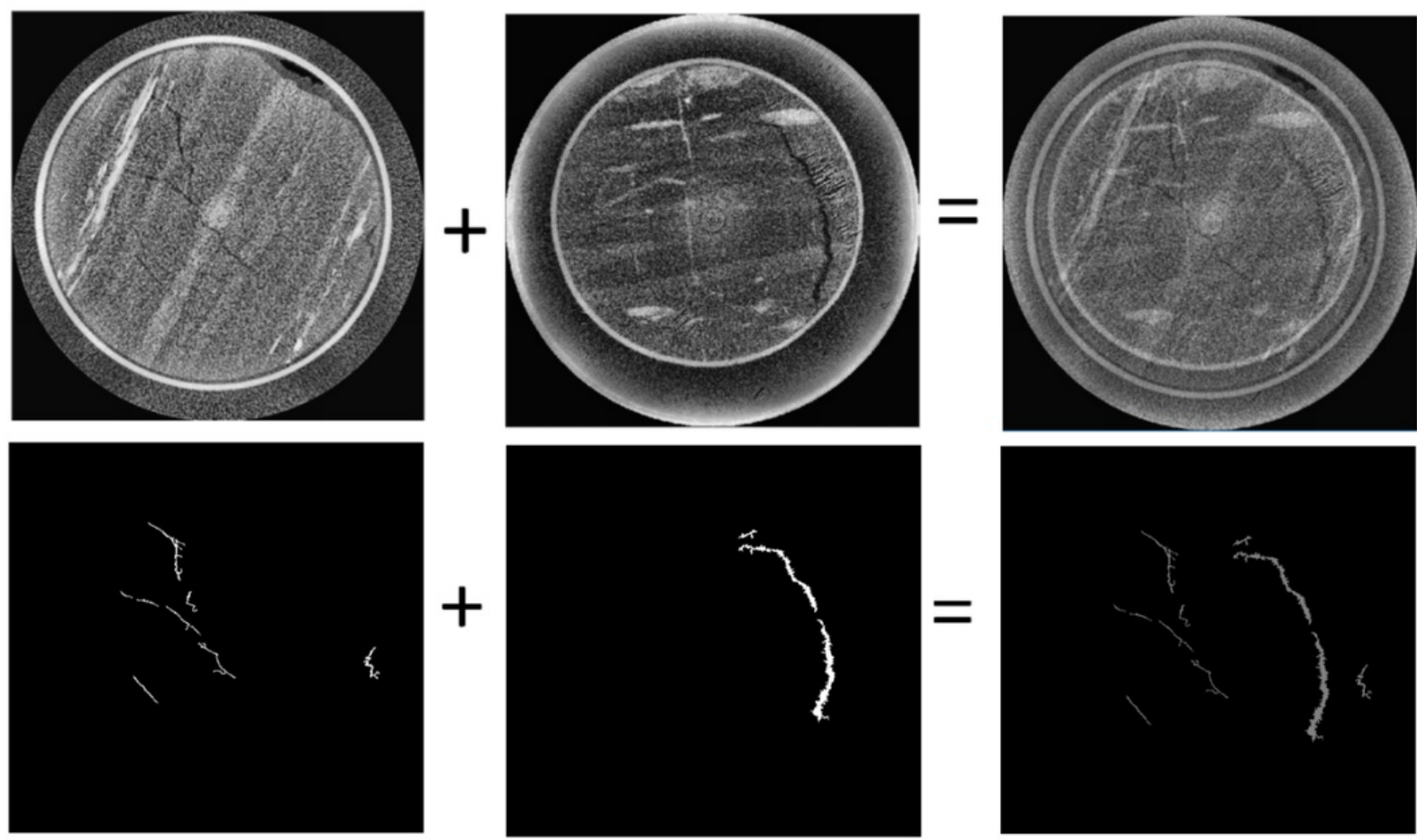

$$
\left(f_{0}(x)\right)
$$

$\left(f_{1}(x)\right)$

$(g(x))$

Figure 6

Image( ) generated by image( ) and image ( ) after linear superposition operation withweights of $\beta=0.5$.
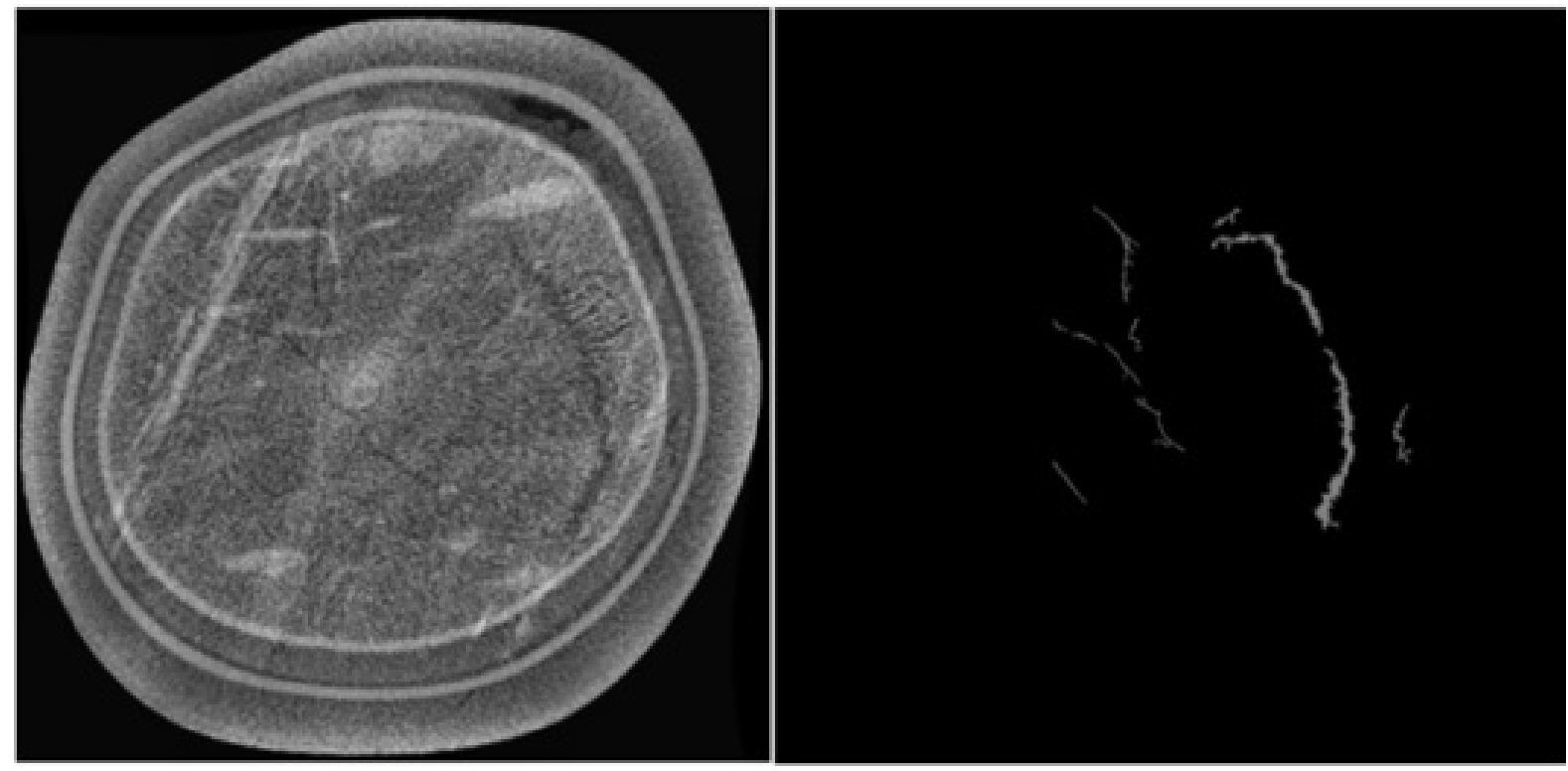

Figure 7

Examples of result after elastic distortion. 


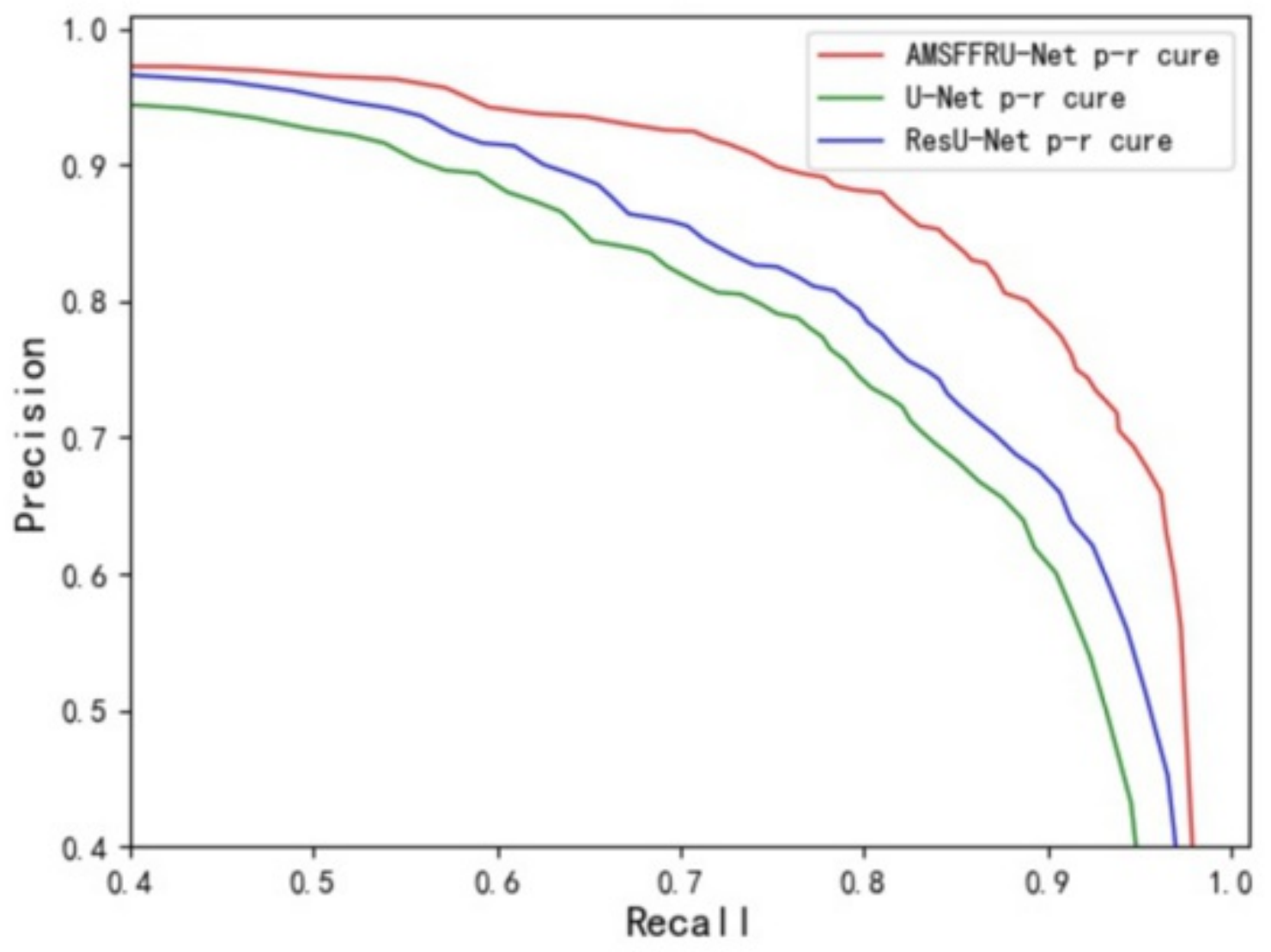

Figure 8

Precision-Recall curves for various methods on our test dataset of coal rock CT images. 

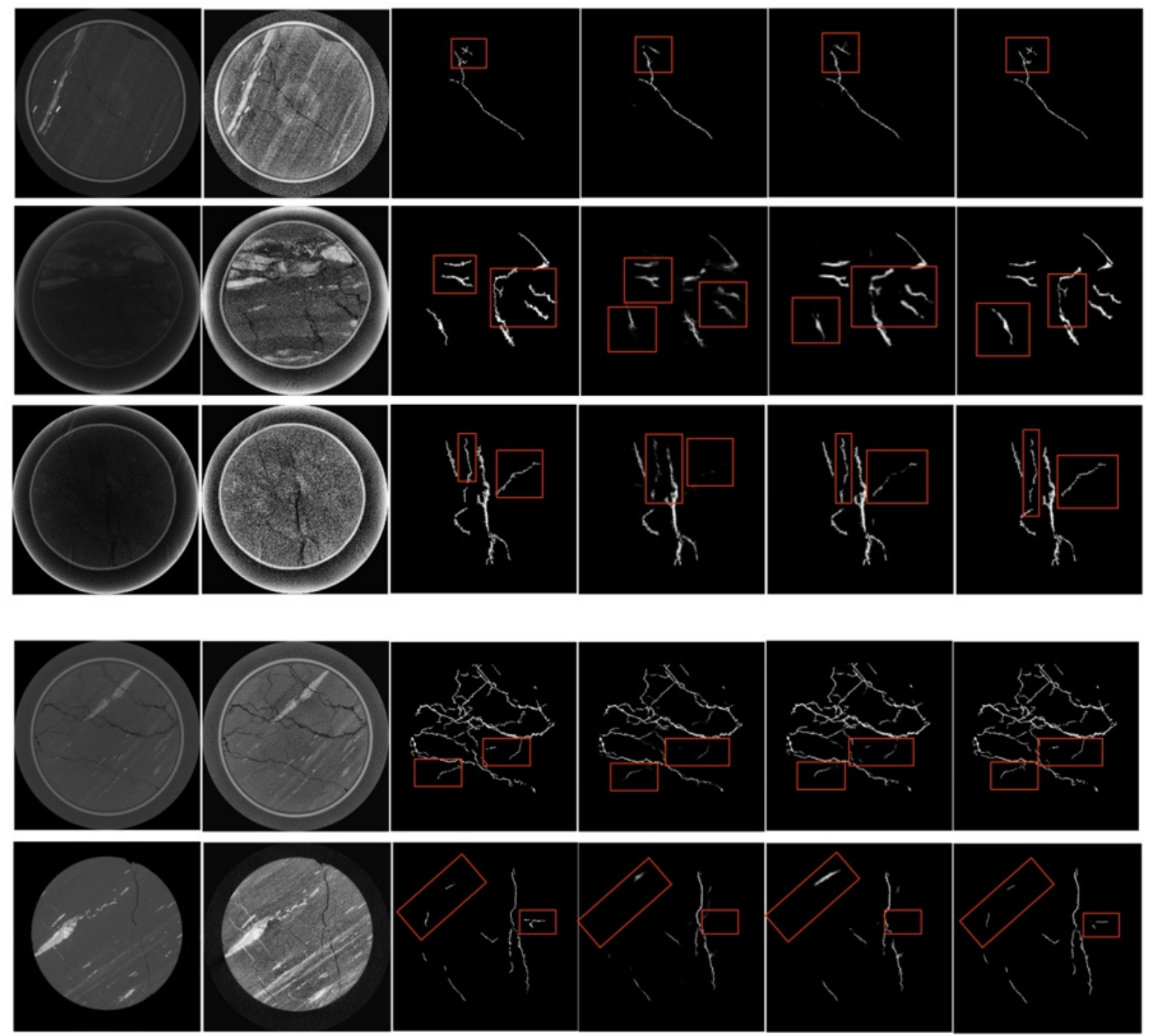

(a)

(b)

(c)

(d)

(e)

(f)

Figure 9

Comparisons of fracture segmentation results on our test dataset, which Contains 5 different textures of coal:(a)Original image.(b)Preprocessed image.(c)Ground truth.(d)U-net.(e)ResUnet.(f)We proposed AMSFFRU-net. 